\title{
A Homozygous Contiguous Gene Deletion in Chromosome 16p13.3 Leads to Autosomal Recessive Osteopetrosis in a Jordanian Patient
}

\author{
Alessandra Pangrazio • Annalisa Frattini - Roberto Valli $\cdot$ Emanuela Maserati \\ Lucia Susani · Paolo Vezzoni · Anna Villa $\cdot$ Waleed Al-Herz $\cdot$ Cristina Sobacchi
}

Received: 17 April 2012 / Accepted: 26 June 2012/Published online: 31 July 2012

(C) Springer Science+Business Media, LLC 2012

\begin{abstract}
Human malignant autosomal recessive osteopetrosis (ARO) is a genetically heterogeneous disorder caused by reduced bone resorption by osteoclasts. Mutations in the CLCN7 gene are responsible not only for a substantial portion of ARO patients but also for other forms of osteopetrosis characterized by different severity and inheritance. The lack of a clear genotype/phenotype correlation makes genetic counseling a tricky issue for CLCN7-dependent osteopetrosis. Here, we characterize the first homozygous interstitial deletion in 16p13.3, detected by array comparative genomic hybridization in an ARO patient of Jordanian origin. The deletion involved other genes besides $C L C N 7$, while the proband displayed a classic ARO phenotype; however, her early death did not allow more extensive clinical investigations.
\end{abstract}

The authors have stated that they have no conflict of interest.

A. Pangrazio · A. Frattini - L. Susani · P. Vezzoni ·

A. Villa $\cdot$ C. Sobacchi

UOS/IRGB, Milan Unit, CNR, Milan, Italy

A. Pangrazio $\cdot$ L. Susani $\cdot$ P. Vezzoni $\cdot$ A. Villa

C. Sobacchi $(\square)$

Humanitas Clinical and Research Center, via Manzoni 56,

20089 Rozzano, Milan, Italy

e-mail: cristina.sobacchi@humanitasresearch.it

A. Frattini $\cdot$ R. Valli $\cdot$ E. Maserati

Department of Experimental and Clinical Medicine,

University of Insubria, Varese, Italy

W. Al-Herz

Pediatric Department, Faculty of Medicine,

Kuwait University, Kuwait City, Kuwait

W. Al-Herz

Allergy and Clinical Immunology Unit, Pediatric Department,

Al-Sabah Hospital, Kuwait City, Kuwait
The identification of this novel genomic deletion involving a large part of the CLCN7 gene is of clinical relevance, especially in prenatal diagnosis, and suggests the possibility that this kind of mutation has been underestimated so far. These data highlight the need for alternative approaches to genetic analysis also in other ARO-causative genes.

Keywords Osteopetrosis - CLCN7 - Deletion · a-CGH · Diagnosis

Autosomal recessive osteopetrosis (ARO) is a rare inherited disease characterized by increased bone density. Since the original identification of the first gene involved in its pathogenesis, our view of the genetics of human ARO has become much more complicated: at least six genes (TCIRG1, CLCN7, OSTM1, PLEKHM1, RANKL, and $R A N K)$ can be affected, and each gene presents a wide spectrum of mutations with very few cases of recurrent changes [1]. Recently, the identification of two large genomic deletions in the TCIRG1 gene [2,3] has highlighted the limits of standard methods for mutational analysis and the possibility that similar mutations could be present also in other known ARO genes.

In the last decade array comparative genomic hybridization (a-CGH) has become widely used in clinical practice as it allows the detection of submicroscopic chromosome imbalanced aberrations, which can be responsible for several pathologic conditions [4].

Here, we describe a homozygous contiguous gene deletion in chromosome 16p13.3 found using this approach. The deletion affects 19 out of 25 exons in the CLCN7 gene and two additional genes and is responsible for the disease in a Jordanian ARO patient. 


\section{Materials and Methods}

Samples

DNA samples were obtained from the patient and her parents after receiving informed consent. Genomic DNA was extracted from peripheral blood lymphocytes by standard techniques (overnight incubation in $1 \times$ Tris EDTA/0.4 \% SDS/10 mg/mL proteinase K). The investigation was approved by the Comitato Etico Indipendente Istituto Clinico Humanitas IRCCS.

\section{TCIRG1 and CLCN7 Gene Mutation Analysis}

Each gene was studied by individual amplification and direct automated sequencing of exons and intron-exon boundaries as previously described $[3,5]$.

\section{Array $\mathrm{CGH}$}

Array $\mathrm{CGH}$ (a-CGH) was performed on an Agilent microarray platform (Santa Clara, CA). The 4x44K wholegenome microarray slides were custom-designed using Agilent's eArray 5.0 online software (http://www.agilent. com) enriched with 5,467 probes for the CLCN7 and TCIRG1 gene regions, with an average probe spacing of 125 bp. Sample preparation, labelling, and microarray hybridization were performed according to Agilent $\mathrm{CGH}$ Enzymatic Protocol version 6.2.1. Slides were acquired using the Agilent G2565CA scanner and analyzed using Agilent Feature Extraction 9.5.1 software. The a-CGH profile was extrapolated using Agilent Genomic Workbench 6.5.0.18 software.

\section{Characterization of the Deletion}

The deletion was characterized by PCR analysis and direct sequencing using a forward primer in intron 3 of the $\mathrm{N}$ acetylglucosamine-1-phosphate transferase, gamma subunit $($ GNPTG) gene (5'-AGGGGTGAGTGGAATGTCAG-3') and a reverse primer in intron 6 of the $C L C N 7$ gene $\left(5^{\prime}-\mathrm{AA}\right.$ CCTCAGGAGGAGCAGACA-3' ${ }^{\prime}$ ).

\section{Results and Discussion}

The patient was born from consanguineous parents of Arabic descent. She presented soon after birth with increased bone density on X-ray (Fig. 1), severe anemia requiring transfusions, pancytopenia, hepatosplenomegaly, failure to thrive, and chronic diarrhea due to infections and pseudomembranous colitis, suggesting a partial, secondary immune deficit; however, lymphocyte subsets in the

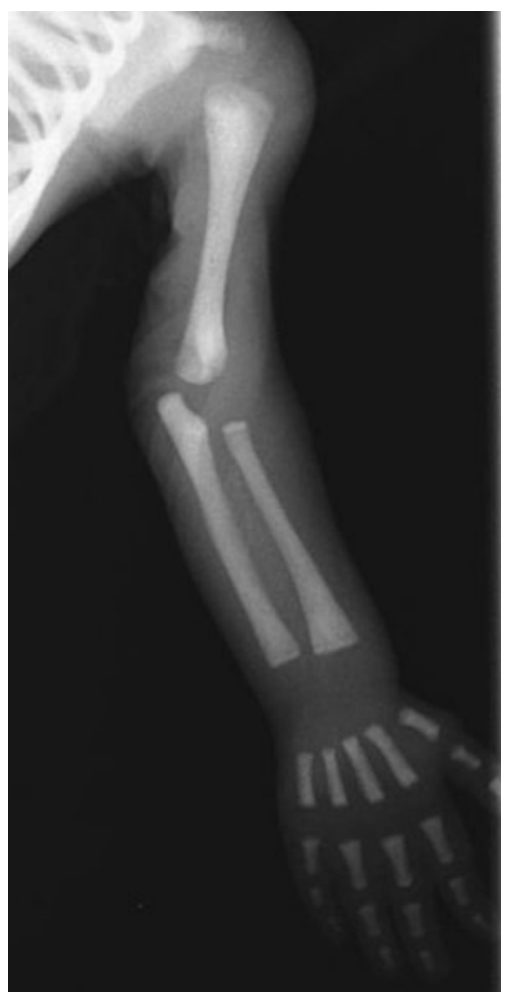

Fig. 1 X-ray of the left arm of the proband, demonstrating the osteopetrotic phenotype

periphery were within the normal range, and marked thrombocytopenia did not allow further investigations. Laboratory analysis showed hypophosphatemia, hypocalcemia, and congenital hypothyroidism, which was treated with L-thyroxine (25 $\mu \mathrm{g}$ daily).

Ophthalmoscopy revealed pale optic disks and macula scarring of both eyes, while the optic nerve canals were symmetric and within normal limits at a CT scan performed at 4 months of age.

The severe condition of the patient did not allow further investigation; she eventually died of sepsis at 6 months of age. No autopsy was performed.

The patient was investigated for mutations in the TCIRG1 gene and subsequently in CLCN7 since these two genes contribute to about $65 \%$ of ARO patients. No mutation was found in the TCIRG1 gene, while lack of amplification of exons 7-25 of the CLCN7 gene in the patient raised the possibility of the presence of a large homozygous genomic deletion, possibly extending beyond the gene itself. a-CGH identified a deletion on chromosome $16 \mathrm{p} 13.3$, involving the CLCN7 gene. A large number of probes showed a highly negative fluorescence $\log$ ratio $(-1$ to $-4 /-\infty)$, highlighting homozygous deletion for the entire affected region [6]. The proximal breakpoint of the deletion was mapped between probes A_16_P57375613 and A_16_57375614 localized at $1,408,559$ and $1,409,150 \mathrm{bp}$, respectively, while the distal breakpoint was between probes A_16_33816661 and 
A_16_3381666 at 1,509,177 and 1,509,209 bp, respectively (hg19 assembly) (Fig. 2).

The deletion encompassed several genes: exons 4-11 of GNPTG, the entire coding sequence of the unkempt homolog (Drosophila)-like (UNKL) gene, exons 7-25 of $C L C N 7$, and additional regions not fully characterized (C16orf91 and CCDC154) (Fig. 3a). Subsequently, in order to exactly define the breakpoints, a series of forward and reverse primers were designed on the basis of the genomic localization of the a-CGH probes flanking the deletion. A PCR product of 1,350 bp was obtained only in the patient and in her parents but not in a healthy control, using a forward primer in intron 3 of the GNPTG gene and a reverse primer in intron 6 of the CLCN7 gene. Direct sequencing of the amplicon allowed us to determine the exact size of the deletion, which was $101,621 \mathrm{bp}$ (CLCN7:c.594+193_oGNPTG:c.178+6298; the mutation nomenclature conforms to www.hgvs.org/mutnomen).

The presence of a chimeric RNA or of protein products deriving from the truncated genes (CLCN7 and GNPTG) could not be investigated due to the lack of an appropriate sample from the patient (only DNA was available).

In order to unravel the molecular mechanisms responsible for this large genomic deletion, we performed in silico analysis (http://repeatmasker.org) and identified repeated sequences within $100 \mathrm{bp}$ from the breakpoints, namely, a LINE element in intron 3 of the GNPTG gene and a simple repeat in intron 6 of the CLCN7 gene (Fig. 3b), possibly involved in the recombination event.
The short arm of chromosome 16 has been extensively studied since it contains a large number of genes. It is often involved in pathological and nonpathological rearrangements, and other interstitial deletions have been reported in the heterozygous state [7-9]. In some cases CLCN7 is involved, too [7, 10]; however, to our knowledge none of them has been associated with an osteopetrotic phenotype, even though heterozygous mutations in CLCN7 are responsible for autosomal dominant osteopetrosis type II (ADOII, MIM 166600). This further confirms our previous suggestion [5] that heterozygous null mutations do not interfere with normal bone resorption and that haploinsufficiency is not causative for ADOII. In agreement with this hypothesis, the proband's parents did not report any symptom possibly related to osteopetrosis.

One of the genes involved in the deletion herein described, GNPTG, is known to cause mucolipidosis III gamma (MLIII $\gamma$, MIM 252605), a slowly progressive lysosomal storage disorder characterized by short stature, cardiomegaly, developmental delay, and skeletal abnormalities. In early childhood, X-rays usually reveal dysostosis multiplex, which worsens with age, giving rise to generalized osteopenia. The proband did not display any obvious clinical feature related to MLIII $\gamma$; however, the severity of the osteopetrotic phenotype and the early death due to secondary immunodeficiency could have overcome mucolipidosis manifestations.

Regarding the $U N K L$ gene, so far there is no evidence of its involvement in pathological conditions. Preliminary in
Fig. 2 Array CGH profile for $16 \mathrm{p} 13.3$ enriched region. The deletion is shown by the software as light green bars as a negative gap of probe fluorescence $\log$ ratios (between -1 and $-4 /-\infty)$. The position of the probes identifying the proximal and distal breakpoints is shown by arrows

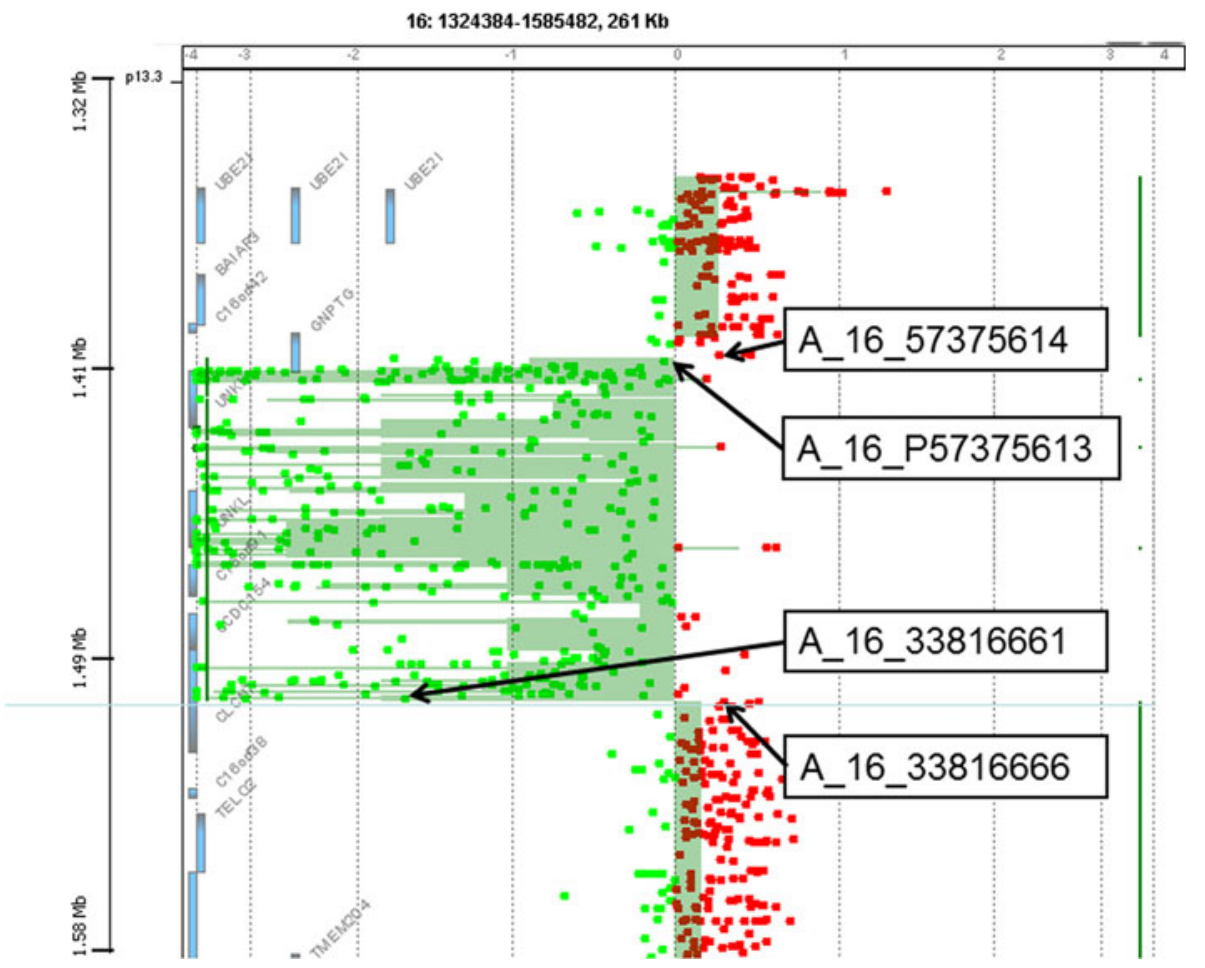


A

Chromosome: 16; Location: 16p13.3

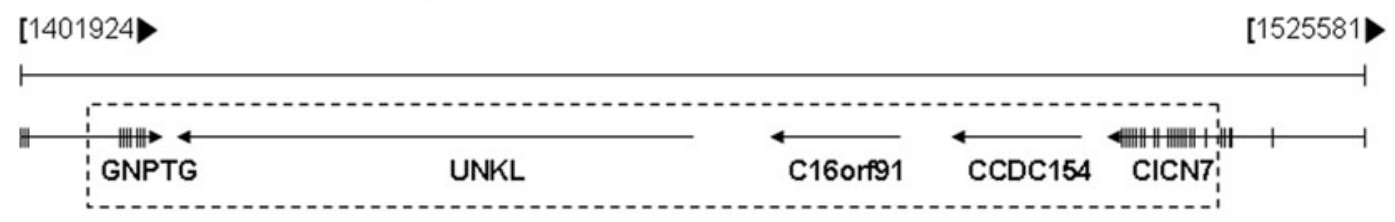

B

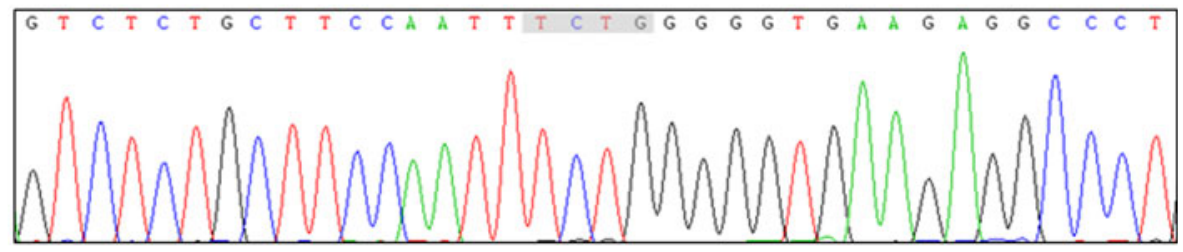

\begin{tabular}{|c|c|c|}
\hline Breakpoint & Position & Sequence \\
\hline Distal & $1408555-1408658$ & ct gtgaacttgggt gtaast att tgagtctct gettccaate tetg agagtaGCTT TCC TGGATC TTA TTAGAA TTCCAT GTT TAGCTT TTT GAGG \\
\hline & & 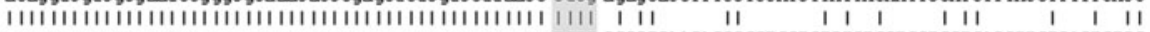 \\
\hline Junction & & ataggct get gtgaacttgggt gtaaat att gagtetct gettccaatt TCTG GGGGTGAAGAGGCCCT GGT GTGTCT CCTCCTCCT GAGGIT GTGAGTCTGG \\
\hline & & 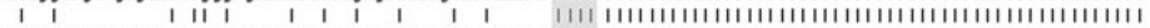 \\
\hline Proximal & $1510177-1510280$ & gtgaggg TGCCCAGCCCTCTCC TGCTAGGTCACGACACCACCCCAGCGC TCTC GGGCTGAAGAGGCCCTGGTGTGTCT CCTCCTCCTGAGTT TTGAGTCTGG \\
\hline
\end{tabular}

Fig. 3 Characterization of the deletion. a Schematic representation of the chromosomal region with the genes involved in the deletion here depicted by the dashed rectangle. For GNPTG and CLCN7 genes the intron-exon structure is shown. b Upper panel Sequence of the amplicon; the precise breakpoint lies in the 4 bp highlighted in gray and shared by both intron 3 of the GNPTG gene and intron 6 of
CLCN7. Lower panel Sequence of breakpoint junctions aligned to the reference sequences. The position of distal and proximal reference comes from hg19 assembly; repeats located within $100 \mathrm{bp}$ from the breakpoints are shown in lowercase. The regions of homology where recombination may have occurred are highlighted in gray

give rise to a wide range of clinical pictures, even in the same family. Although monoallelic, dominant "benign" and biallelic "severe" recessive osteopetrosis are usually distinct, several patients with intermediate severity have been described [5, 13]. In this regard, the recognition of nonobvious heterozygous deletions could shed further light on this disorder. In addition, it is extremely relevant for genetic counseling of family members and, in particular, for a correct prenatal diagnosis; indeed, failure to detect this type of mutation can lead to the misinterpretation of the single variant sequence identified, which can be erroneously considered as a homozygous mutation when masked by a coincident exonic deletion.

Recent findings $[2,3]$ together with the present data suggest that large genomic deletions could be underestimated, and adequate approaches of genetic screening should be developed. This is particularly relevant for osteopetrosis, in which the results of molecular analysis have a significant impact on patient management.

Acknowledgments The technical assistance of Dario Strina and of Giuseppe Montalbano is acknowledged. This work was partially supported by Fondazione Cariplo (Grant 2008-2218 to C. S.), the Telethon Foundation (Grant GGP10116 to C. S.), the PRIN Project (200999KRFW-002 to P. V.), Giovani Ricercatori from Ministero della Salute (Grant GR-2008-1134625 to C. S.), and Progetto Premiale CNR Invecchiamento. 


\section{References}

1. Villa A, Guerrini MM, Cassani B, Pangrazio A, Sobacchi C (2009) Infantile malignant, autosomal recessive osteopetrosis: the rich and the poor. Calcif Tissue Int 84:1-12

2. Pangrazio A, Caldana ME, Sobacchi C, Panaroni C, Susani L, Mihci E, Cavaliere ML, Giliani S, Villa A, Frattini A (2009) Characterization of a novel Alu-Alu recombination-mediated genomic deletion in the TCIRG1 gene in five osteopetrotic patients. J Bone Miner Res 24:162-167

3. Pangrazio A, Caldana ME, Lo Iacono N, Mantero S, Vezzoni P, Villa A, Sobacchi C (2012) Autosomal recessive osteopetrosis: report of 41 novel mutations in the TCIRG1 gene and diagnostic implications. Osteoporos Int. doi:10.1007/s00198-011-1878-5

4. Shinawi M, Cheung SW (2008) The array CGH and its clinical applications. Drug Discov Today 13:760-770

5. Pangrazio A, Pusch M, Caldana E, Frattini A, Lanino E, Tamhankar PM, Phadke S, Lopez AG, Orchard P, Mihci E, Abinun M, Wright M, Vettenranta K, Bariae I, Melis D, Tezcan I, Baumann C, Locatelli F, Zecca M, Horwitz E, Mansour LS, Van Roij M, Vezzoni P, Villa A, Sobacchi C (2010) Molecular and clinical heterogeneity in CLCN7-dependent osteopetrosis: report of 20 novel mutations. Hum Mutat 31:E1071-E1080

6. Valli R, Maserati E, Marletta C, Pressato B, Lo Curto F, Pasquali F (2011) Evaluating chromosomal mosaicism by array comparative genomic hybridization in hematological malignancies: the proposal of a formula. Cancer Genet 204:216-218

7. Daniels RJ, Peden JF, Lloyd C, Horsley SW, Clark K, Tufarelli C, Kearney L, Buckle VJ, Doggett NA, Flint J, Higgs DR (2001)
Sequence, structure and pathology of the fully annotated terminal $2 \mathrm{Mb}$ of the short arm of human chromosome 16. Hum Mol Genet 10:339-352

8. Nelson M, Quinonez S, Ackley T, Iyer RK, Innis JW (2011) Multiple congenital anomalies and developmental delay in a boy associated with a de novo 16p13.3 deletion. Am J Med Genet A 155A:612-617

9. Mohd Fadley MA, Ismail A, Keong TM, Yusoff NM, Zakaria Z (2012) Chromosomal 16p microdeletion in Rubinstein-Taybi syndrome detected by oligonucleotide-based array comparative genomic hybridization: a case report. J Med Case Rep 6:30

10. Harteveld CL, Kriek M, Bijlsma EK, Erjavec Z, Balak D, Phylipsen M, Voskamp A, di Capua E, White SJ, Giordano PC (2007) Refinement of the genetic cause of ATR-16. Hum Genet 122:283-292

11. Lorès P, Visvikis O, Luna R, Lemichez E, Gacon G (2010) The $\mathrm{SWI} / \mathrm{SNF}$ protein BAF60b is ubiquitinated through a signalling process involving Rac GTPase and the RING finger protein Unkempt. FEBS J 277:1453-1464

12. Rastogi MV, LaFranchi SH (2010) Congenital hypothyroidism. Orphanet J Rare Dis 5:17

13. Frattini A, Pangrazio A, Susani L, Sobacchi C, Mirolo M, Abinun M, Andolina M, Flanagan A, Horwitz EM, Mihci E, Notarangelo LD, Ramenghi U, Teti A, Van Hove J, Vujic D, Young T, Albertini A, Orchard PJ, Vezzoni P, Villa A (2003) Chloride channel CLCN7 mutations are responsible for severe recessive, dominant, and intermediate osteopetrosis. J Bone Miner Res 18: 1740-1747 